\title{
Neues Antihistaminikum macht Furore
}

\begin{abstract}
llergiker können aufatmen - der neue, gut verträgliche H1-Rezeptorantagonist Levocetirizin, das aktive Enantiomer des Razemats Cetirizin, steht seit Anfang letzten Jahres zur Verfügung. Auch Kinder ab einem Körpergewicht von $30 \mathrm{~kg}$ können damit behandelt werden. Experten waren sich auf einem Symposium anlässlich der Rheinischen Gespräche („RheinGAU 2001“) einig, dass diese Substanz bei allergischer Rhinitis wirksamer ist als Cetirizin.

C. DeVos (Brüssel) hob das besondere pharmakologische Profil - hohe Absorptionsrate, fast 100\%ige Bioverfügbarkeit und lineare Pharmakokinetik - des neuen Antiallergikums hervor. Die Wirkung von $5 \mathrm{mg}$ Levocetirizin, $5 \mathrm{mg}$ Dextrocetirizin, $10 \mathrm{mg}$ Cetirizin sowie Plazebo auf Histamin-induzierte Nasensymptome erklärte L. Klimek (Wiesbaden), indem er die Ergebnisse einer Studie von D.-Y. Wang und Mitarbeitern (2001) präsentierte: Levocetirizin und Cetirizin führten im Gegensatz zu Plazebo zu einer Halbierung des maximalen Histamin-induzierten Anstiegs des nasalen Widerstands und zu einer vierfachen Erhöhung des Histamin-Schwellenwertes von 8 auf $32 \mathrm{mg} / \mathrm{ml}$. Niesen wurde in den beiden Verumgruppen unter Levocetirizin und Cetirizin ebenfalls signifikant reduziert.
\end{abstract}

\section{Überzeugende Resultate bei sai- sonaler und perennialer Rhinitis}

In einer Studie von Horak und Mitarbeitern wurden 35 Allergiker in der Wiener Provokationskammer an zwei Tagen je sechs Stunden lang mit Hausstaubmilben Allergen-provoziert. Dabei linderte Levocetirizin den komplexen Symptomscore aus Niesen, Nasenlaufen und Juckreiz schneller als das Vergleichspräparat Loratadin. Unter Levocetirizin lag die Ansprechrate - Patienten mit mindestens 20\%iger Symptomreduktion - bei etwa $84 \%$ und war im Vergleich mit Placebo hochsignifikant besser $(\mathrm{p}=$ 0,005).

Die Wirksamkeit bei saisonaler allergischer Rhinitis wurde an 470 Patienten über zwei Wochen geprüft. Die Resultate zeigten, dass 2,5, 5 bzw.
10 mg Levocetirizin täglich die Symptome an Augen und Nase deutlich reduzierten. Dabei kristallisierte sich $5 \mathrm{mg}$ Levocetirizin als die Dosis mit dem besten Risiko-Nutzen-Verhältnis heraus.

Symposium und Pressegespräch beim „RheinGAU 2001“, Assmannshausen, 2001. Veranstalter: UCB Pharma

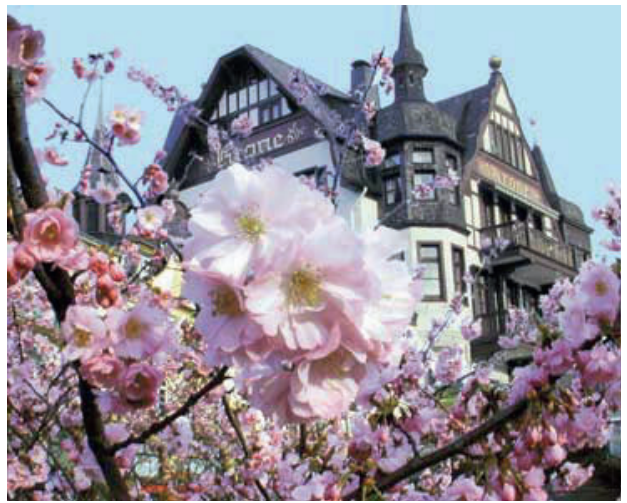

Bei der RheinGAU-Tagung in Assmannshausen diskutierten Deutschlands Allergologen über aktuelle Erkenntnisse der Allergie-Prävention, -Diagnose und -Therapie.

\section{Orale Entzündungshemmung mit hoher Patientenakzeptanz}

$T_{\text {th }}^{\text {h }}$ herapeutische Basis in der Asthmatherapie ist die Entzündungshemmung. Als Standardmedikamente hierfür werden derzeit inhalative Kortikoide eingesetzt. Sie hemmen die inflammatorischen Vorgänge in ihren frühen Stadien, sind jedoch nicht in der Lage, die Leukotriensynthese adäquat $\mathrm{zu}$ hemmen. „Die Leukotriene sind jedoch entscheidend für viele Asthmasymptome", so W. Petro (Bad Reichenhall).

Leukotriene greifen in einer späteren Phase der Asthmakaskade ein und sind mitverantwortlich für Bronchokonstriktion, Mukushypersekretion sowie bronchiale Hyperreaktivität und locken Entzündungszellen in das $\mathrm{Ge}$ webe. Mit den nunmehr seit rund drei Jahren zur Verfügung stehenden Leukotrien-Rezeptorantagonisten lassen sich diese pathophysiologischen Wirkungen unterbinden.

So blockiert der Wirkstoff Montelukast durch seine spezifische Bindung am Cys-LT1-Rezeptor das Andocken der Leukotriene an diesen Rezeptor und wirkt dadurch antientzündlich, bronchialerweiternd und schleimbildungshemmend. Die Zahl der Eosinophilen im Blut als auch im induzierten Sputum wird unter Montelukast in vergleichbarem Umfang wie unter Beclometason vermindert. Der Wirkeintritt des Leukotrien-Rezeptorantagonisten erfolgt deutlich früher als bei den inhalativen Steroiden.
Aufgrund dieser Eigenschaften ist Montelukast gut geeignet, Kortikoide einzusparen. In Fällen von leichtem bis mittelschweren Asthma, vor allem aber beim Belastungsasthma, kann auch schon die alleinige Montelukastgabe ausreichend sein. Das Antiasthmatikum ist bei uns erhältlich unter dem Warenzeichen Singulair ${ }^{\circledR}$ (Tablette mit $10 \mathrm{mg}$ für Erwachsene), Singulair ${ }^{\circledR}$ junior (Kautablette mit $5 \mathrm{mg}$ für Schulkinder und Jugendliche) sowie Singulair ${ }^{\circledR}$ mini (Kautablette mit $4 \mathrm{mg}$ für Kleinkinder ab 2 Jahren).

\section{Einmal abends auf der Bettkante}

Die Compliance ist bei diesem oral einzunehmenden Entzündungshemmer im Vergleich zu den inhalativen Medikamenten sehr gut. Der einfache Einnahmemodus - „einmal abends auf der Bettkante" - und die vom Patienten deutlich spürbare Verminderung der Bronchokonstriktion und der Hypersekretion führen zu einer hohen Patientenakzeptanz. Erfahrungsgemäß, so Petro, lässt sich diese Akzeptanz noch einmal steigern, indem man das Antiasthmatikum den Patienten als „kortisonfreies Kortison" erklärt. $\quad b k$

Pressekonferenz „Asthmatherapie zwischen Theorie und Praxis - Maßgeschneidert von Anfang an“, Westerland/Sylt, 2001. Veranstalter: MSD Sharp \& Dohme 